\title{
Silicon nanocrystal memories
}

\author{
Fakhrosadat Rastegari $^{1^{*}}$ and Foroghsadat Rastegari ${ }^{2}$ \\ ${ }^{1}$ Department of Electricity, Yazd branch, Islamic Azad University of Yazd, Yazd, Iran \\ ${ }^{2}$ Member of Young Researchers Club, Yazd branch, Islamic Azad University, Yazd, Iran \\ frastegari@yahoo.com*
}

\begin{abstract}
In this paper, we present an overview of silicon nanocrystals memories. Silicon nanocrystals are as storage nodes in these memories. These devices show promising characteristics as candidates for future deep-submicron non-volatile memories. The structure and fabrication of these devices are explained at first. Then the size, density, and emission of silicon nanocrystals in nanocrystal memory device are considered. Finally, a FinFET silicon nanocrystal memory is introduced as a practical sample.
\end{abstract}

Keywords: Silicon nanocrystal, Memory, Storage, FinFET Introduction

In this paper, it is presented an overview of memory structures fabricated by using silicon nanocrystals as storage nodes. These devices show promising characteristics as candidates for future deep-submicron non-volatile memories. The finite probability of having a cluster of two or three defects in the tunnel oxide, producing a huge local increase of the tunnel current (namely SILC), determines the presence of bits with an anomalous failure, which are widely recognized as the most important concern in Flash devices (Fig. 1). The amplitude of the SILC increases when the tunnel oxide is thinned, limiting the oxide scaling.

Fig 1. Schematic of a nanocrystal memory cell

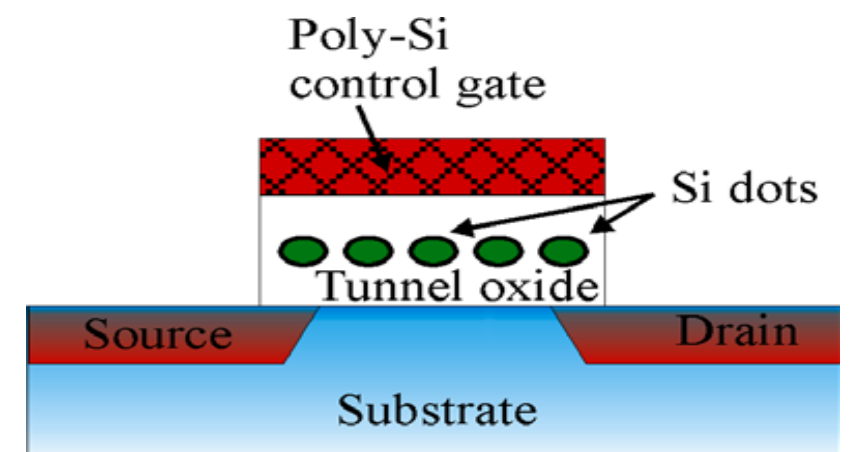

The use of discrete-trap storage nodes in conventional Flash memory technology has been invoked as one of the key items for existing Flash memory survival. The very special effect of the localized trapping is the high reliability associated with it. In fact, in DiscreteTrap memories a single leakage path due to a defect (intrinsic or SILC-related) in the oxide can only discharge a single storage node (Lombardo \& De Salvo, 2004). Some different type of memory-cell structures, employing discrete-trap type storage nodes, has been demonstrated in the literature.

Silicon nanocrystal dot memory

Nonvolatile memory devices using nc-Si-dot-based electrically isolated charge-storage nodes in an oxide film as a floating gate have emerged in the last decade

(Fig.1), where charge leakages through localized oxide defects could be greatly reduced, therefore, improving performance reliabilities, as well as the memory-retention time. Furthermore, a much thinner tunneling oxide film in nanodot memory devices causes a major tunneling mechanism due to direct tunneling hence enabling a faster write/erase speed compared to Fowler-Nordheim tunneling which is met in conventional flash type memory devices (Oda and Huang, 2007). Charge injection and storage in dense arrays of $\mathrm{nc}-\mathrm{Si}$ dots in $\mathrm{SiO}_{2}$ is a key aspect of their performance. The ultimate goal for this class of devices is approaching the few- or singleelectron storage in a small number of nc-Si elements at room temperature, relying on the Coulomb blockade effects as a new electron transport principle, commonly referred to as single electron memory.

The high-density integration capability and low-power consumption enable them to be an attractive candidate for the next generation digital nonvolatile memory applications. However, in spite of the successful demonstrations of memory operations based on singleelectron transports, the charge retention time of nc-Si dot memory devices is too short for practical nonvolatile memory applications. The improved retention time was demonstrated to be possible without any significant loss of programming speed based on the modifications of floating-gates by the dual memory nodes. Charge retention characteristics reveal how charges are stored in nc-Si memory nodes. A major essential issue for nc-Si memory devices in nonvolatile memory applications is the data retention characteristics over extended period. After some electrons are trapped, at a chosen reading voltage (e.g., flat-band voltage), the stored electrons have a finite probability to tunnel back to the drain, which could cause a gradual shift of channel current or capacitance of $\mathrm{SiO}_{2} / \mathrm{nc}-\mathrm{Si} / \mathrm{SiO}_{2}$ diode.

These gradual shifts reflect barrier heights/widths, internal electric fields, defect or interfacial states, and so on. Therefore, investigations of time dependences of memory windows as well as charging/discharging behaviors of nc-Si dots correlated with Coulomb blockade 
and quantum confinement effects offer better understanding of charge retention characteristics of nc-Si memory devices. Investigation of charging and discharging in nc-Si dots, based on measurements of capacitance-voltage and conductance-voltage characteristics, shows that interface states and deep defects correspond to charging and discharging processes. However, at low defect and interfacial state density, electron charge/discharge is only dominated by bounding electrons in nc-Si dots. For a $\mathrm{SiO}_{2} / \mathrm{nc}-\mathrm{Si} / \mathrm{SiO}_{2}$ capacitor memory device, in time-dependent capacitance measurements, the memory-retention time was found to exceed $5 \mathrm{~h}$ (calculated time for the loss of about $10 \%$ of the original charge) at room temperature (Oda \& Huang, 2007).

Instead of being governed by deep defects, at low defect and interfacial state density, electron charge/discharge is only dominated by electron bounded in nc-Si dots. A repulsive "built-in" electric field from nc-Si dots to silicon substrate created and controlled by charge loss in nc-Si dots was proposed to explain such long-term retention time. On the other hand, a longer retention time can be achieved by introducing a certain number of deep trapping centers in nc-Si dots with decreasing the interfacial states at the tunneling-oxide/Si interface. Compared with pure nc-Si floating-gate memory devices, nitrided nc-Si-dot-based memory devices were experimentally demonstrated to be helpful to remarkably increase retention time by three orders of magnitude, as shown in Fig. 2, without sacrificing write/erase time, in which memory operations based on combined charge/discharge processes of nitrided nc-Si dot systems. The stored charges in such memory nodes were

Fig 2. Time dependence of the stored-charge from the flatband state, after electrons were injected in floating-gates, shows a logarithmic discharging behavior. The gate voltage was kept at the initial flat-band voltage: $-1.6 \mathrm{~V}$ for sample $A$ (surface nitrided nc-Si dots) and -2.4V for sample B (without nitridation). The inset shows the structure of memory device.

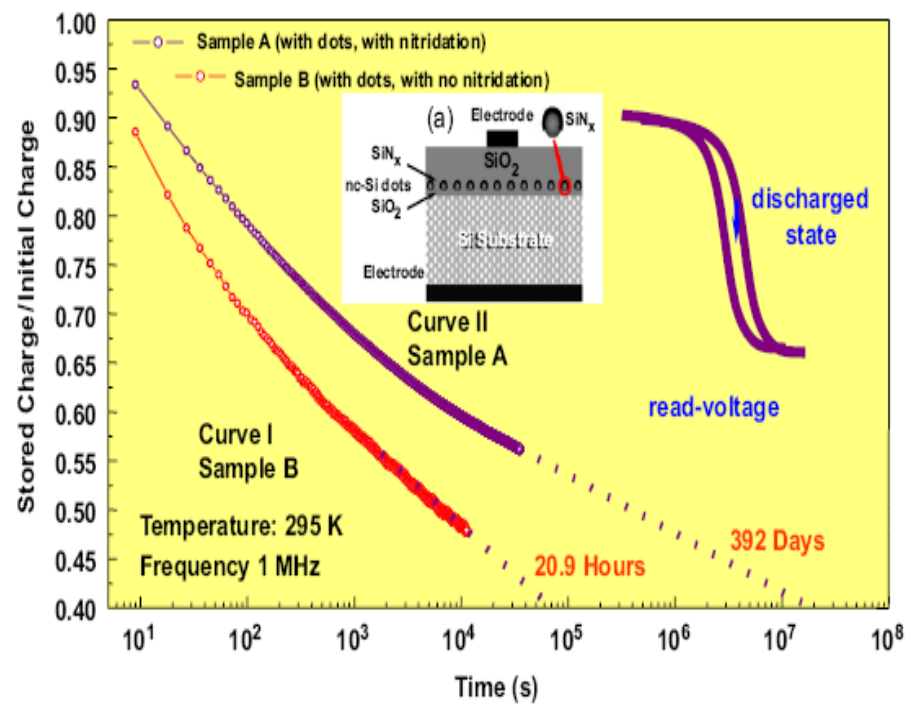

identified not only in nc-Si dots but also in defect-states of silicon-nitride films, corresponding to electron delocalized and localized states, respectively.

Materials and device fabrication

It should be stated that the key technology for silicon nanocrystals memories is how to fabricate the nanometer scale dots. In fact, high dot density, nanometer size, good uniformity in size and shape, lateral isolation, planarity on the tunnel oxide, background charge minimization are all required in a reliable fabrication process. Several methods to synthesize the $\mathrm{Si}$ dots have been proposed and investigated in the last years: ion implantation (Gebel et al., 2001), aerosol (De Blauwe et al., 2000), and chemical vapor deposition (CVD).

Nanocrystalline silicon (nc-Si) particles with size less than $10 \mathrm{~nm}$ were prepared by VHF plasma-enhanced decomposition of silane gas. Pulsed gas plasma processing, in which the nucleation and the growth period were controlled precisely, turned out to be effective for the preparation of monodispersed nc-Si particles.

The size and density of silicon nanocrystals in nanocrystal memory device

Structural characterization via transmission electron microscopy and atomic force microscopy of arrays of small $\mathrm{Si}$ nanocrystals embedded in $\mathrm{SiO}_{2}$, important to many device applications, is usually difficult and fails to correctly resolve nanocrystal size and density. It is demonstrated that scanning tunneling microscopy (STM) imaging enables a much more accurate measurement of the ensemble size distribution and array density for small $\mathrm{Si}$ nanocrystals in $\mathrm{SiO}_{2}$. The reflection high-energy electron diffraction pattern further verifies the existence of nanocrystallites in $\mathrm{SiO}_{2}$. The present STM results enable nanocrystal-charging characteristics to be more clearly understood: It find the nanocrystal charging measurements to be consistent with single charge storage on individual $\mathrm{Si}$ nanocrystals. Both electron tunneling and hole tunneling processes are suggested to explain the asymmetric charging/discharging processes as a function of bias (Gebel et al., 2001; De Blauwe et al., 2000).

To fully exploit their potential advantages over conventional floating gate memory, it is essential to control as accurately as possible $\mathrm{Si}$ nanocrystal size, depth distribution, and areal density, as well as nanocrystal surfacepassivation and oxide defect density in $\mathrm{SiO}_{2}$ matrix, all in a process compatible with ultra-largescale integration. Transmission electron microscopy (TEM) is the most widely used tool to characterize nanocrystal size and distribution with high resolution. It has used a combination of contact-mode atomic force microscopy (AFM) and reflection high-energy electron diffraction (RHEED) to identify the existence of nanocrystals, and used an ultrahigh vacuum scanning tunneling microscope (UHV STM) to estimate nanocrystal size and aerial density (Feng \& Yu, 2005). 
Fig 3. Structure of all silicon-based light emitting device, which consists of nc-Si active layer with quasi-direct radioactive recombination, nc-Si high efficiency electron emitter for excitation, and three-dimensional photonic crystal layer for optical confinement
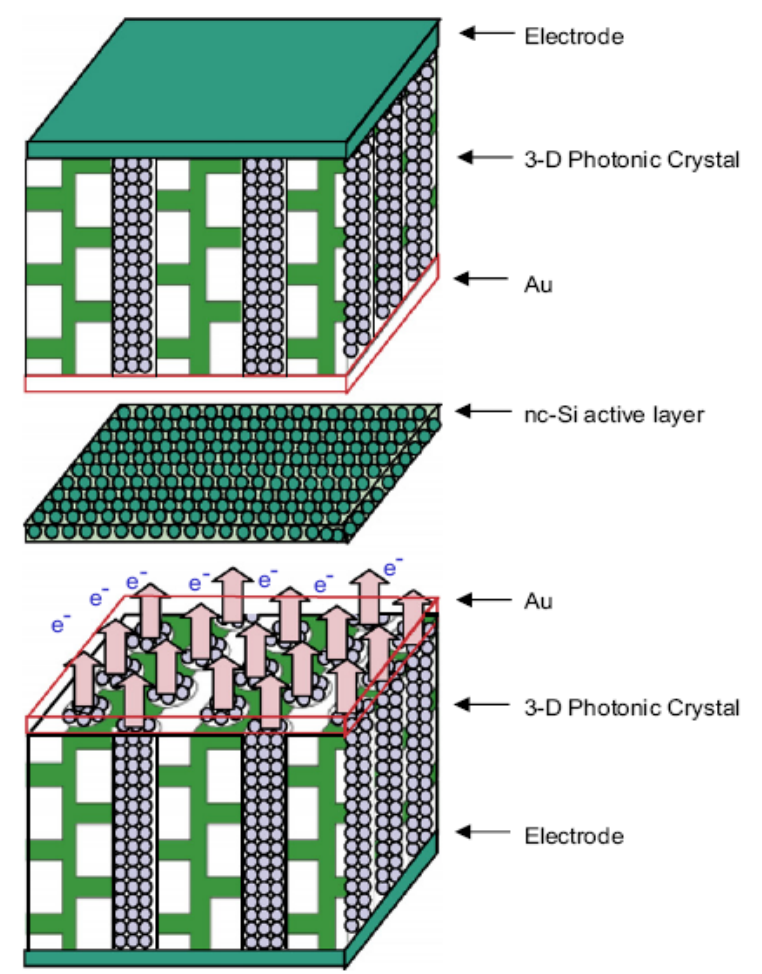

To evaluate quantitative data like nanocrystal sizes and density, STM is found to be a better tool. Some investigations have used STM to characterize CdSe and InAs quantum dots on Au (Millo et al., 2000). The STM measurements of Si nanocrystals fabricated through lowpressure chemical vapor deposition and nanocrystalline silicon films obtained by boron implantation of amorphous Si layers have also been reported.

Charge storage and electron/light emission properties of silicon nanocrystals

Monodispersed silicon nanocrystals show novel electrical and optical characteristics of silicon quantum dots, such as single-electron tunneling, ballistic electron transport, visible photoluminescence, and high-efficiency electron emission. Electrical properties of nc-Si particles were investigated by employing nanoscale electrodes, both planar and vertical configurations, prepared by electron-beam lithography.

Single-electron memory effects were studied using a short-channel MOSFET having Si quantum dots as a floating gate. Storing of electrons in individual Si dots was evaluated by Kelvin probe force microscopy (KFM). It was proposed novel memory devices based on nano electromechanical systems (Tsuchiya et al., 2004).

Photoluminescence and electron emission were observed from surface-oxidized nc-Si particles. Efficiency
Vol. 5 No. S3 (Mar 2012)

ISSN: 0974- 6846

of the no-phonon-assisted transition increases with decreasing core Si size. Electron emission efficiency as high as $5 \%$ has been achieved for the Sinanocrystalbased cold electron emitter devices. The nonMaxwellian energy distribution of emitted electrons suggests that the mechanism of electron emission is due to ballistic transport through arrays of surface-oxidized $\mathrm{Si}$ nanocrystals. Combined with the ballistic electron emission, the quasi-direct light emission properties can be used for developing Si-based lasers.

Silicon photonic devices

The discovery of optical gain in $\mathrm{nc}-\mathrm{Si}$, triggered widespread research for silicon-based lasers. Stimulated emission was reported for a nanostructured silicon pn junction diode using current injection. Silicon-based Raman lasers have been demonstrated with pulsed optical pumping and continuous wave operation (Pavesi et al., 2000).

Silicon-based laser operation is expected as a key technology in realizing opto-electronic integrated circuits. Silicon based lasers operate only under very high excitation conditions, making it difficult to incorporate them into the CMOS circuits in which ultra low power consumptions are required.

A completely different approach is proposed to realize silicon-based laser operation using threedimensional photonic crystal structures combined with ncSi quantum dots as light and electron emitters, as shown in Fig.3. First, the three-dimensional photonic crystal structures are introduced to increase the stimulated emission probability caused by the standing wave at the photonic band edge, thereby resulting in a significant increase in the external quantum efficiency. Second, ncSi quantum dots are used for constructing an active layer, which emits light in the visible band due to the quantum confinement effect.

Nanocrystalline silicon was fabricated with uniform diameter of $8 \mathrm{~nm}$ using pulsed gas plasma CVD method. A high-efficiency visible photoluminescence was observed for the surface-oxidized nc-Si indicating the quantum confinement effect and quasi-direct recombination. Another key feature of device is that the dimension of nc-Si can be controlled simply by changing the thermal oxidation time. This is vital to control the electron emission properties, the luminescence wavelength of $\mathrm{nc}-\mathrm{Si}$, the refractive index of the structured nc-Si arrays, and therefore to optimize the photonic band gap properties of the whole device.

Nanocrystal Memory Devices Characterization using the Charge Pumping Technique

The results are presented here by performing the two level charge pumping (CP) technique on nanocrystal memory devices. The charge pumping method allows the determination of the silicon dots characteristics such as their density, their spatial distribution within the dielectric and their effective diameter (Masson \& Militaru, 2002). 
Memory-cell structures employing discrete-trap type storage nodes, thus operating with a small finite number of electrons, have recently attracted much attention as very promising scalable ultra-dense low power memories, capable of exceeding the performance limits of conventional floating gate devices in terms of write/erase speed, endurance, and refresh time. Because of its very high sensitivity, the charge pumping technique is an excellent tool to determine the characteristics of the traps within the dielectric of MOS transistors. Experimental results and simulations demonstrate that the response of the silicon dots does not correspond to a trap-like behavior but it is closer to a floating gate like behavior, confirming results of previous works.

Moreover, the use of the CP technique with a square gate pulse allows the determination of the spatial distribution of the dots within the dielectric, the extraction of the effective surface of the dots and an estimation of the silicon dots density. The determination of the distribution of the Si-dot density as a function of the effective diameter, Ddot(Deff), can be used to evaluate the effective shape repartition of the dots for a given process.

The fin field-effect transistor (FET) silicon nanocrystal floating gate memory

The FinFET silicon nanocrystal floating gate memory with a gate length of $100 \mathrm{~nm}$ was successfully fabricated and it revealed a memory effect as well as a suppressed short-channel effect (Soo et al., 2006). The photo-CVD for silicon nanocrystal formation and the plasma doping method for formation of source-drain extension (SDE) were proposed for fabrication of FinFET nanocrystal floating gate memory devices. The Si nanocrystals were formed over all channel area, including the vertical $\mathrm{Si}$ fin surface by photo-CVD. The plasma doping is effective for the $3 \mathrm{D}$ architecture of FinFET device and reduces the complexity of the ion doping process. The fabricated FinFET nanocrystal flash memory device showed better sub threshold swing and DIBL characteristics than bulk-Si devices, and it revealed a memory effect as well as a suppressed short channel effect. Further optimizations of tunnel oxide and control oxide can improve the performance of FinFET nanocrystal memory device with a larger $\mathrm{V}^{\text {th }}$ window as well as a higher integrity.

Conclusion

Silicon nanocrystals with novel functions such as charge and energy quantization and efficient photon/electron emission are promising for future electronics applications. Silicon nanodot memory device can be a promising alternative for high-density nonvolatile memory. The significance and useful application of this device in electronics need more research studies.

\section{References}

1. De Blauwe J, Ostraat M, Green ML, Weber G, Sorsch T, Kerber A, Klemens F, Cirelli R, Ferry E, Grazul JL, Baumann F, Kim Y, Mansfield W, Bude J, Lee JTC, Hillenius SJ, Flagan RC and Atwater HA (2000) A
Vol. 5 No. S3 (Mar 2012) ISSN: 0974-6846

Novel Aerosol-Nanocrystal Floating-Gate Device For Nonvolatile Memory Applications. Technical Digest of the International Electron Devices Meeting, IEEE.

2. Feng $\mathrm{T}$ and $\mathrm{Yu} \mathrm{H}$ (2005) Probing the size and density of silicon nanocrystals in nanocrystal memory device applications. Appl. Phys. Lett. 86, 033103(1)033103(3).

3. Gebel $T$, Rebohle L, Zhao J, Borchert $D$, Fröb $H$, Borany JV and Skorupa W (2001) lon beam synthesis based formation of Ge-rich thermally grown $\mathrm{SiO}_{2}$ layers:a promising approach for a silicon based light emitter. Mater. Res. Soc. Symp. Proc. 638, F18.1.1.

4. Lombardo S and De Salvo B (2004) Silicon nanocrystal memories. Microelectron. Eng. 72, 388394.

5. Masson $P$ and Militaru L (2002) NanoCrystal memory devices characterization using charge pumping technique, ESSDERC 2002, pp.235-238.

6. Millo O, Katz D, Cao YW and Banin U (2000) Single Electron Tunneling Through Single InAs Nanocrystal Quantum Dots. Phys. Rev. B. 61, 16773-16777.

7. Oda S and Huang SY (2007) Charge storage and electron/light emission properties of silicon nanocrystals. Physica E. 38, 59-66.

8. Pavesi L, Dal Negro L, Mazzoleni C, Franzo G and Priolo $F(2000)$ Optical gain in silicon nanocrystals. Nature. 408, 440-444.

9. Soo S, Cho W, Ahn C, Im K, Yang J, Baek I, Lee S, Koeng SL (2006) Fabrication of fin field-effect transistor silicon nanocrystal floating gate memory using photochemical vapor deposition. Appl. Phys. Lett. 88, 223502.

10. Tsuchiya $\mathrm{Y}$, Takai $\mathrm{K}$, Momo N, Yamaguchi $\mathrm{S}$, Shimada T, Koyama S, Takashima K, Higo Y, Mizuta $\mathrm{H}$ and Oda S (2004) High-speed and Nonvolatile Nano Electromechanical Memory incorporating Si Quantum Dots" IEEE Silicon Nanoelectronics Workshop, Honolulu. 